ing formula,

$$
\left(e^{-\lambda / R}+e^{-2 \lambda / R}+e^{-3 \lambda / R}+\cdots\right) \frac{\left(-\alpha+\frac{1}{l_{1}}\right) \lambda}{(\alpha-\lambda) \frac{1}{l_{1}}}
$$

So, we can estimate more precisely the ratio as follows

Delayed neutron background Maximum neutron density

$$
=\left(1+\frac{\mathrm{e}^{-\lambda / R}}{1-\mathrm{e}^{-\lambda / R}}\right) \frac{\left(-\alpha+\frac{1}{l_{1}}\right) \lambda}{(\alpha-\lambda) \frac{1}{l_{1}}} .
$$

which leads to

$$
=\left(1+\frac{\mathrm{e}^{-\lambda / R}}{1-\mathrm{e}^{-\lambda / R}}\right)^{\frac{\lambda}{\alpha}}
$$

for

$\alpha \ll \frac{1}{l_{1}}$.

\title{
研案諭文
}

\section{On the Distribution of the Slowing-down Time of Neutrons}

中性子減速時間の分布

\author{
By Takashi NAKAYAMA*
}

\begin{abstract}
The distribution of the slowing-down time of neutrons in an infinite homogeneous medium has been determined by the stochastic method. It is found that the distribution approaches that of gamma type asymptotically for a sufficiently large lethargy.

The experimental results are compared with theoretical curve, and a good agreement have been obtained.
\end{abstract}

\section{Introduction}

For simplicity let us assume that neutrons travel in an infinite homogeneous medium consisting of identical atomic nuclei of mass number $M$, that there is no absorption in the medium, and that the scattering cross section is independent of the neutron energy. Let $g(t, u) d t$ be the probability that the slowingdown time required for a neutron to reach a lethargy higher than $u$ lies in the interval $(t, t+d t)$. The basic equation obeyed by the probability density $g(t, u)$ is mentioned in Pál and Németh's paper ${ }^{(1)}$;

$$
\begin{aligned}
\frac{\partial g(t, u)}{\partial t}= & -\frac{v_{0}}{\lambda_{s}} \int_{0}^{u} \mathrm{e}^{-u^{\prime} / 2}\left\{1-\int_{0}^{u-u^{\prime}} \tau\left(u^{\prime \prime}\right) d u^{\prime \prime}\right\} \\
& \times \frac{\partial g\left(t, u^{\prime}\right)}{\partial u^{\prime}} d u^{\prime},
\end{aligned}
$$

where $\lambda_{s}$ is the mean free path for scattering, $v_{0}$ the velocity of pulsed neutrons with lethargy 0 , and $w(u) d u$ the probability that the lethargy increment of a neutron in a collision lies in the interval $(u, u+d u)$, i.e.,

$$
\begin{aligned}
& w(u)=\frac{1}{1-\alpha} \mathrm{e}^{-u}, \quad \text { if } 0 \leqq u \leqq \ln \frac{1}{\alpha},(2) \\
& w(u)=0, \quad \text { if } u>\ln \frac{1}{\alpha} \text { or } u<0,
\end{aligned}
$$

while

$$
\alpha=\left(\frac{M-1}{M+1}\right)^{2} .
$$

Here we shall explain the meaning of Eq. (1) in detail. The probability that a neutron with lethargy $u$ will collide with the atom in the time interval $(t, t+d t)$ is

* 中山 隆。Reactor Phys. Div., Japan Atomic Energy Res. Inst. (日本原于力研然所 原子炬物理 部) 


$$
\frac{v}{\lambda_{s}} d t=\frac{v_{0}}{\lambda_{s}} \mathrm{e}^{-u / 2} d t .
$$

The probability that a neutron with lethargy $u^{\prime}$ collides with the atom in the medium and its lethargy after collision exceeds $u$ is

$$
1-\int_{0}^{u-u} z(u) d u^{\prime \prime}
$$

Let $f(t, u) d t d u$ be the probability that in the time interval $(t, t+d t)$ a neutron's lethargy lies in the interval $(u, u+d u)$, then

$$
\begin{aligned}
& g(t, u) d t=d t \int_{u t}^{\infty} f\left(t, u^{\prime}\right) d u^{\prime} \\
& f(t, u)=-\frac{\partial g(t, u)}{\partial u} .
\end{aligned}
$$

By thinking collectively the above facts, we can show that the right hand side of Eq. (1) is the probability that a neutron collides with the atom in the time interval $(t, t+d t)$ and its lethargy exceeds $u$ for the first time, and this probability equals to $\partial g(t, u) / \partial t$ which is the left of Eq. (1).

\section{Solution of the Basic Equation}

We have to remark that on the right hand side of Eq. (1), the expression $\left(\partial / \partial u^{\prime}\right) \cdot g\left(t, u^{\prime}\right)$ has a $\delta$-function-like singularity taking into account the initial condition. To avoid this difficulty, some particular considerations in the derivation of the function $g(t, u)$ are involved.

Let be $h(t, u) d u$ the probability that the neutron lethargy at time $t$ lies in the interval $(u, u+d u)$, assuming its lethargy was zero at time $t=0$.

It is now easy to see that

$$
g(t, u)=\frac{v_{0}}{\lambda_{s}} \int_{0}^{u} \mathrm{e}^{-u^{\prime} / 2}\left\{1-\int_{0}^{u-w(u} w\left(u^{\prime \prime}\right) d u^{\prime \prime}\right\} h\left(t, u^{\prime}\right) d u^{\prime}
$$

while $h(t, u)$ satisfies the integro-differential equation

$$
\begin{aligned}
\frac{\partial h(t, u)}{\partial t}= & -\frac{v_{0}}{\lambda_{s}} \mathrm{e}^{-u / 2} h(t, u) \\
& +\frac{v_{0}}{\lambda_{s}} \int_{0}^{u} \mathrm{e}^{-u^{\prime} / 2} h\left(t, u^{\prime}\right) w\left(u-u^{\prime}\right) d u^{\prime}
\end{aligned}
$$

with the initial condition

$$
h(0, u)=\delta(u) \text {. }
$$

Let us introduce the notation

$$
t_{n}(u)=\int_{0}^{\infty} t^{n} g(t, u) d t, \quad \Theta_{n}(u)=\int_{0}^{\infty} t^{n} h(t, u) d t .
$$

Considering Eqs. (6) and (7), we obtain from Eqs. (4) and (5)

$$
\begin{gathered}
t_{n}(u)=\frac{v_{0}}{\lambda_{8}} \int_{0}^{u} \mathrm{e}^{-u^{\prime} / 2}\left\{1-\int_{0}^{u-u u^{\prime}} z\left(u^{\prime \prime}\right) d u^{\prime \prime}\right\} \Theta_{n}\left(u^{\prime}\right) d u^{\prime} \\
n \Theta_{n-1}(u)=\frac{v_{0}}{\lambda_{s}} \mathrm{e}^{-u / 2} \Theta_{n}(u) \\
-\frac{v_{0}}{\lambda_{8}} \int_{0}^{u} \mathrm{e}^{-u^{\prime} / 2} w\left(u-u^{\prime}\right) \Theta_{n}\left(u^{\prime}\right) d u^{\prime} \\
\quad(n \geqq 1)
\end{gathered}
$$

while

$$
\begin{aligned}
\delta(u)= & \frac{v_{0}}{\lambda_{s}} \mathrm{e}^{-u / 2} \Theta_{0}(u) \\
& -\frac{v_{0}}{\lambda_{s}} \int_{0}^{u} \mathrm{e}^{-u^{\prime} / 2} w\left(u-u^{\prime}\right) \Theta_{0}\left(u^{\prime}\right) d u^{\prime} .
\end{aligned}
$$

Denoting the Laplace transforms of $t_{n}(u)$ and $\Theta_{n}(u)$ by

$$
L_{n}(s)=\int_{0}^{\infty} \mathrm{e}^{-s u} t_{n}(u) d u, \quad \chi_{n}(s)=\int_{0}^{\infty} \mathrm{e}^{-s u} \Theta_{n}(u) d u
$$

a simple calculation shows that

$$
L_{n}(s)=n !\left(\frac{\lambda_{s}}{v_{0}}\right)^{n} \frac{1}{s} \prod_{j=1}^{n} \frac{1}{1-W(s-j / 2)}
$$

where $W(s)=\int_{0}^{\infty} \mathrm{e}^{-s u} w(u) d u$.

\section{Slowing-Down in Hydrogen}

In the case of slowing-down of neutrons in hydrogen as a moderator,

$$
w(u)=\mathrm{e}^{-u}
$$

and we have

$$
W(s)=\frac{1}{s+1}
$$

Substituting Eq. (12) into (11), we have

$$
L_{n}(s)=n !\left(\frac{\lambda_{s}}{v_{0}}\right)^{n} \frac{1}{s} \prod_{j=1}^{n} \frac{s-j / 2+1}{s-j / 2} .
$$

$t_{n}(u)$ being a monotonically increasing function of its argument, according to the theorem

$$
\lim _{u \rightarrow \infty} e^{-\pi u / 2} t_{n}(u)=\lim _{s \rightarrow 0} s L_{n}(s+n / 2)
$$

and Eq. (13), the asymptotic expression

$$
t_{n}(u) \sim(n+1) !\left(\frac{\lambda_{s}}{v_{0}}\right)^{n} \mathrm{e}^{n u / 2}
$$

can be derived for $t_{n}(u)$ with high lethargy values. By the moment theorem ${ }^{(2)}$, the probability distribution whose moments are represented by Eq.(15) is the gamma type distribution and its probability density function, $g(t, u)$, is 


$$
g(t, u)=a^{2} t \mathrm{e}^{-a t}
$$

where

$$
a=\frac{v_{0}}{\lambda_{s}} \mathrm{e}^{-u / 2}=\frac{v}{\lambda_{s}} .
$$

\section{Comparison with Experiment}

When the slowing-down time of neutrons in light water is measured by the pulsed neutron technique, neglecting the scattering by oxygen, we proceed as follows; let $X_{1}(t)$, ........, $X_{N}(t)$ be independent random variables which have the identical distribution, that $X_{t}(t)=1$, if the $i$-th neutron's lethargy is greater than $u$ after $t \mu_{\mathrm{sec}}$, and $X_{i}(t)=0$, otherwise, then it is clear that

$$
P\left\{X_{i}(t)=1\right\}=\int_{0}^{t} g(t, u) d t .
$$

Since the total number $X(t)$ of neutrons whose lethargy exceeds $u$ after $t \mu_{\mathrm{sec}}$ is

$$
X(t)=\sum_{i=1}^{N} X_{i}(t),
$$

where $N$ is the total number of pulsed neutrons with initial velocity $v_{0}$. We have the mean value of $X(t)$

$$
E\{X(t)\}=N \int_{0}^{t} g(t, u) d t=N\left\{1-(1+a t) e^{-a t}\right\}
$$

and its variance

$$
V\{X(t)\}=N\left\{1-(1+a t) \mathrm{e}^{-a t}\right\}(1+a t) \mathrm{e}^{-a t}
$$

The comparison of the theoretical with the experimental value is shown in Fig. 1 . It shows good agreement between the curve calculated from Eq. (19) and the experimentally plotted points (the experiment will be reported by Hirakawa, et $a l^{(4)}$ ).

The mean slowing-down time is

$$
t_{1}(u)=2 \frac{\lambda_{s}}{v_{0}} e^{u / 2}=\frac{2}{a} .
$$

From the histogram of experimental slowingdown time, we can get the estimate of $t_{1}(u)$ and thus of $a .\left(a=0.59 \mu \mathrm{sec}^{-1}\right)$ Then the theoretical curve

$$
\int_{0}^{t} g(t, u) d t=1-(1+a t) e^{-a t}
$$

is obtained with use of the value of $a$. The

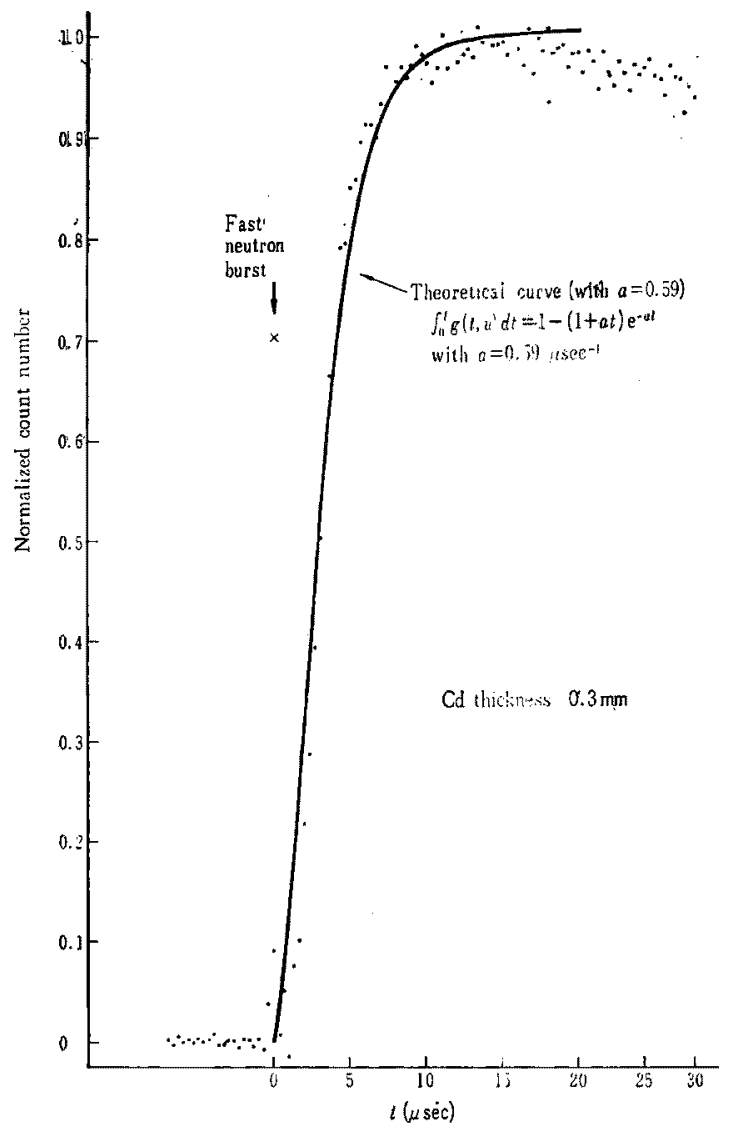

Fig. 1 Slowing-down time distribution of the thermal neutrons detected by the $\mathrm{BF}_{3}$ counter covered with $0.3 \mathrm{~mm}$ thick $\mathrm{Cd}$ sheet

variance of $X(t)$ attains its maximum value $(=0.25)$ at about $3 \mu \mathrm{sec}$ and decreases monotonically as $t$ increases beyond that value. Therefore, it should be noted that larger fluctuation of count number appears at early stage of slowing-down process.

\section{Slowing-down in the Medium OTHER THAN HYDROgEN}

Now we consider the distribution of the slowing-down time of neutrons in the medium other than hydrogen. It can be shown in this case the density function $g(t, u)$ approaches that asymptotically of the gamma type for a sufficiently large $u$ when we adopt the Wigner approximation for the function $z(u)$ from the Eq. (11) as before.

The expression for $g(t, u)$ is 


$$
g(t, u) \sim\left\{\begin{array}{ll}
\frac{a^{\lambda}}{\Gamma(\lambda)} t^{\lambda-1} \mathrm{e}^{-a t} & \text { for } t \geqq 0, \\
0 & \text { for } t<0
\end{array} \text { and for large } u_{\text {s }}\right.
$$

where

$$
a=\frac{v_{0}}{\lambda_{s}} \mathrm{e}^{-u / 2}
$$

and

$$
\begin{aligned}
\lambda & =2 / \xi \\
& =2\left(1+\frac{\alpha}{1-\alpha} \ln \alpha\right)^{-1} .
\end{aligned}
$$

The $n$-th moment, $t_{n}(u)$, of the distribution of the slowing-down time is

$$
t_{n}(u) \sim \frac{\Gamma(\lambda+n)}{\Gamma(\lambda)} a^{-i}
$$

The author wishes to express his gratitude to Prof. R. Nozawa of Tokyo Institute of Technology and Dr. L. Pál of Central Re- search Institute for Physics of Hungary for valuable advice, to Miss $\mathrm{H}$. Hiruta and Miss H. Miyauchi for copy writing and graph tracing.

(Received August 23, 1962)

- REFERENCE

(1) L. PÁL, G. NÉMETH: 'On the Fluctuation of the Slowing-down Time of Neutrons', Nukleonik, 1, 5, 165 167 (1959).

(2) H. Cramer: "Mathematical Methods of Statistics", (1957), Princeton.

(3) S. S. WILKS: "Mathematical Statistics", (1943), Princeton.

(4) N. Hirakawa, T. IjJma, S. Nomoto, T. Nakamura, $H$. Tomioka, M. Nozawa: to be published.

\title{
研究論文
}

\section{The Extraction of Americium and Europium Nitrates by Tri-n-octyl Phosphine Oxide in Toluene}

\author{
硝酸アメリシウムおよび硝酸ユーロピウムのTOPOKよる抽出
}

By Tomitaro ISHIMORI*, Chin Taik RHEE** and Takeo FUJINO*

The extraction behavior of americium and europium was studied in $\mathrm{TOPO}_{*}^{* *}$-nitric acid system. The distribution ratios ( $K_{d}$ values) were measured as respective functions of nitric acid, hydrogen ion and nitrate ion concentrations as well as a function of TOPO concentration.

The obtained curve of $\log K_{d}$ vs. nitric acid concentration exhibited a maximum at $0.25 \mathrm{M}$ nitric acid for both americium and europium. The slope of the plot of log $K_{d}$ vs. logiTOPOlorg. was found to be about 2.5 at TOPO concentrations between 0.5 and $5 \%$. It was also found that the value $K_{d} /(T O P O)^{3}$ org. is scarcely affected by hydrogen ion concentration. At $0.1 \mathrm{M}$ nitric acid, the values of $K_{d} /\left(\mathrm{TOPO}^{3}{ }^{3}\right.$ org. were shown to be nearly third power dependent on the nitrate ion concentration.

These results lend to support the conclusion that only free TOPO extract americium andlor europium. By using this conclusion, the maximal $K_{d}$ value and corresponding acidity were estimated. The peak positions of $K_{d}$ for the extractions of thorium and zirconium were also tried to calculate in this system. These values were satisfactorily consistent with experimental values.

* 石森富太郎, 藤野威男。Chem. Div., Japan Atomic Energy Res. Inst.（日本原子力研究所 化学部)

**, 本鎮沢。Atomic Energy Res. Inst., Korea(韓国 原子力研究所)

** Tri-n-octyl phosphine oxide 